\title{
El risedronato redujo las fracturas de cadera en mujeres ancianas con osteoporosis densitométrica
}

Effect of risedronate on the risk of hip fracture in elderly women. M Mc Clung, P Geusens, P Miller. N Engl J Med, 2001;344:333-340.

Objetivo

Evaluar la eficacia del risedronato en la prevención de fracturas de cadera en mujeres ancianas con osteoporosis o con factores de riesgo distintos de la densidad mineral ósea.

\section{Diseño}

Ensayo clínico aleatorizado multicéntrico, doble ciego y controlado con placebo.

\section{Lugar}

Realizado entre noviembre de 1993 y abril de 1998 en 183 centros de Norteamérica, Nueva Zelanda, Europa y Australia

\section{Pacientes}

Se incluyeron pacientes posmenopáusicas en dos grupos. El primer grupo estaba conformado por mujeres de 70 a 79 años con osteoporosis diagnosticada por densitometría ósea del cuello femoral ( $T$ score menor que -4 o bien menor que -3 más un factor de riesgo). Los factores de riesgo clínicos considerados fueron la dificultad para pararse desde la posición sentada o realizar una marcha en tándem, la injuria debida a una caída en el año anterior a la inclusión, un puntaje psicomotor de Clifton menor o igual a cinco (evalúa la coordinación, con un puntaje de 1 a 12 y un puntaje menor a cinco indica riesgo aumentado de caída), el tabaquismo actual o en los cinco años previos, la historia materna o personal de fractura de cadera y una longitud cadera/axial de $11.1 \mathrm{~cm}$ o más.

El segundo grupo incluyó mujeres mayores de 80 años con, por lo menos, un factor de riesgo no esquelético para fractura de cadera, un score T mayor a -4 o un score T mayor a -3 más una longitud cadera/axial mayor a $11.1 \mathrm{~cm}$

Se excluyeron pacientes con enfermedades importantes, historia reciente de cáncer, enfermedades óseas, resultados de laboratorio alterados, uso reciente de drogas que afecten al hueso, alergia a bifosfonatos, historia de fractura de cadera bilateral o cualquier condición física o mental que impidieran la participación.

\section{Intervención}

Las mujeres de ambos grupos fueron asignadas a recibir durante tres años risedronato $(2.5 \mathrm{mg} /$ día o $5 \mathrm{mg} /$ día) o placebo. Además recibieron $1 \mathrm{~g}$ de carbonato de calcio/día. Las mujeres que presentaban una concentración de vitamina $\mathrm{D}$ en sangre menor a $16 \mathrm{ng} / \mathrm{ml}$ recibieron suplemento de esta vitamina.

\section{Medición de resultados principales}

El punto final primario fue la incidencia de fractura de cadera confirmada radiográficamente. Los puntos finales secundarios fueron la incidencia de fracturas no vertebrales osteoporóticas y la densidad mineral ósea (esta última fue medida sólo en 44 centros)

\section{Resultados principales}

Finalizaron el estudio entre el 60 y $70 \%$ de las pacientes. El análisis fue por intención de tratar. Globalmente, la incidencia de fractura de cadera en el grupo que había recibido risedronato fue de $2.8 \%$, mientras que en el grupo placebo fue $3.9 \%$ (RR: 0.7 ; IC95\%: 0.6 a $0.9 ; p=0.02$ ). En el grupo de mujeres entre 70 y 79 años asignadas a risendronato la incidencia de fracturas fue $1.9 \%$, mientras que en el grupo placebo fue $3.2 \%$ (RR: 0.6; IC95\%: 0.4 a $0.9 ; p=0.009$ ).

No hubo diferencias significativas en el grupo de mujeres de más de 80 años seleccionadas sobre la base de factores de riesgo clínicos. La incidencia de fracturas de cadera en el grupo risedronato fue de $4.2 \%$ y en el grupo placebo, de $5.1 \%(p=0.35)$.

\section{Conclusiones}

El risedronato reduce el riesgo de fractura de cadera en mujeres ancianas con osteoporosis confirmada pero no en las mujeres ancianas seleccionadas sobre la base de factores de riesgo distintos de la densidad mineral ósea.

\section{Comentario}

Este ensayo clínico agrega información de otros estudios previos. 1-2 Demuestra que el risedronato fue efectivo en prevenir la fractura de cadera en mujeres ancianas con osteoporosis diagnosticada con desitometría osea, pero no en mujeres con factores de riesgo clínicos que no tenían valores densitométricos compatibles con osteoporosis.

Es importante tener en cuenta la pérdida considerable de pacientes de los distintos grupos desde el momento de la asignación del tratamiento hasta la finalización del seguimiento, que llegó a ser entre 30 y $40 \%$. Los autores aclaran que las razones de abandono del estudio no fueron diferentes en las ramas de tratamiento con droga activa versus la de intervención con placebo.

Otro aspecto a considerar es que para reclutar mujeres con osteoporosis, se utilizaron T scores menores a -3 y -4 , incluyendo mujeres con grados más severos de osteoporosis que los habitualmente utilizados en la práctica cotidiana (valores de T score menores a -2.5).

Globalmente, se necesitó tratar a 100 (NNT*) mujeres con risedronato durante tres años para prevenir una fractura de cadera. Si dirigimos el análisis a aquellas mujeres de entre 70 y 79 años con osteoporosis diagnosticada por densitometría observamos que el NNT baja a 80; y si hacemos lo propio con aquellas mujeres que habían tenido una fractura vertebral antes de comenzar a tomar risedronato o placebo, advertimos que el NNT desciende hasta 30 Estos distintos NNT reflejan un impacto muy distinto en distintos grupos de mujeres. Estos nos ayudan a "dirigir" la intervención hacia aquella población de mujeres con mayor riesgo de fractura de cadera, lo que redundará en un mayor impacto para las pacientes tratadas, tornando también más razonable la relación entre el costo y el beneficio. 